\title{
An Improved MFD based Regional Traffic Volume Dynamic Control
}

\author{
Yiman Du1a, Jianping $\mathrm{Wu}^{1 \mathrm{~b}}$, Yuhan Jia ${ }^{1 \mathrm{c}}$, Ming $\mathrm{Xu^{1 \textrm {d } }}$
}

\section{(1. Department of Civil Engineering, Tsinghua University, Beijing 100084, China)}

(a. ymducp@gmail.com; b. jianpingwu@tsinghua.edu.cn; c. yhjiathu@126.com; d. xum@bupt.edu.cn; )

\begin{abstract}
The connection between traffic congestion and regional demand has become a consensus. A reasonable road network inflow control is essential to prevent the occurrence of congestion. With a consideration of deployment of detectors, an improved macroscopic fundamental diagram-based traffic volume dynamic control method via the feedback control is proposed in this study. Based on OD distribution of trips and connectivity of nodes, a novel method is adopted to identify the key nodes, on which the detectors are laid. And Artificial Neural Network is adopted to predict traffic volume of those sections without detectors. As a case study, the proposed methodology is applied to the regional Road network which is located in downtown area of Nanning, China. Adequate survey and analysis are carried out under current road traffic conditions via simulation study. It is proved that the regional traffic volume dynamic control can ensure steady and orderly regional traffic flow, and enhance the mobility in a saturated traffic conditions.
\end{abstract}

Keywords: regional traffic volume dynamic control; macroscopic fundamental diagram; feedback control; microscopic traffic simulation

\section{Background}

"Urban area represents the part with densest social-economic and land development activities in the built-up area [1]". Due to the functional characteristics of urban area, an increasing number of cities are facing a sharp contradiction between traffic and development. Massive traffic congestions have become a headache in most cities of China. Whether from the occurrence frequency, intensity, or from the affected area, the overall impact of the traffic congestion on urban development is

1 DOI reference number: 10.18293/SEKE2016-038 increasingly evident.

Typically traffic congestion can be controlled either by increasing road capacity, or reducing traffic demand. From a practical point of view, it's impossible to solve the problem by unlimited expansion of construction of new transport infrastructure. Therefore, it's becoming more important to take better traffic management measures to scientifically regulate traffic flow so as to alleviate traffic congestion and improve urban mobility with the limited resources.

The connection between traffic congestion and regional demand has become a consensus after significant amount of research. When traffic flow reaches the upper limit of the road capacity, congestion occurs. DAGANZO C.F. in 1996 [2] proposed a continuous model of merge operation, which described the process of gradual self-destruction of traffic flow without outside intervention in urban expressway. His study also revealed that the collapse process can be reversed by restricting the ratio of mainline input flow below a critical level. Andre D.P. [3] studied the relation between traffic congestion and traffic equilibrium theory by analyzing traffic congestion during peak periods. The study showed that in commuting fast lane during a peak period, traffic congestion was mainly due to the reach of upper limit of the traffic flow. Therefore, a reasonable road network inflow control is the core to prevent the occurrence of gridlock. For urban areas, traffic volumes control in central region is an effective method to ensure smooth traffic flow.

Traffic volume control means minimizing the number of participants in traffic, shortening the operation time, and reducing road area occupied by participants. When the traffic load in urban road network is close to saturation or saturated, there is no room for the transfer of traffic pressure. Thus traffic volume control has become a good option. Staggered shifts, Congestion charging and vehicle 
license plates restriction rules are the commonly used regional traffic volume control measures. These measures control traffic volume in urban network via policies and regulations. However, the three measures do involve not only traffic problems, but also trade-offs for every participator, and will even affect the public interest then easily lead to disputes. Therefore, transportation managers are more willing to dynamically adjust traffic loads in urban centers from a technical point of view.

A lot of research has been done on signal control. However, isolated or arterial signal control can only partially improve the isolated intersection situation, but cannot cope with large-scale traffic congestion. Many scholars dynamically divided traffic areas into multiple sub-regions for optimal control, and then achieved coordinated control of the entire region [4]. In addition, signal control based on multi-agent technology is also an important area of regional signal control research, and $\mathrm{Li}$ Zhenlong[5], Wiering[6], Zhang Hui[ 7] etc. used modeling and algorithms of coordination of urban traffic signal control based on agent technology to solve the regional coordinated problems and the entire urban traffic network optimization problems. Some scholars also introduced fuzzy logic, neural networks etc. to regional traffic signal control [8]. Existing regional signal control system can improve the overall operating efficiency of road network under unsaturated traffic conditions, but it does not work under the heavy traffic conditions. And at present, there is still no ripe system control program put into use at home and abroad under heavy traffic conditions [9]. A report by the U.S. Federal Highway Administration Commission noted that there was no signal optimization tool which was able to effectively deal with the situation of traffic congestion [10].Based on the assumption that all links are detector-equipped the fundamental network diagram was exploited by Mehdi etc. to improve mobility in saturated traffic conditions via feedback gating control using microscopic simulation [11]. It is easy to obtain the appropriate information of each link by simulation. However, it is unrealistic to distribute the detectors on every link of road network due to the limitation of funding and complexity of maintenance. Therefore, location and numbers of detectors are important. Based on this, with the consideration of deployment of detectors, an improved macroscopic fundamental diagram- based traffic volume dynamic control method via the feedback control is proposed in this study. A novel method based on OD distribution of trips and connectivity of nodes is adopted to identify the key nodes, on which the detectors are laid. And Artificial Neural Network are adopted to predict traffic volume of those sections without detectors. As a case study, the proposed methodology is applied to the regional Road network which is located in downtown area of Nanning. Adequate survey and analysis are carried out under current road traffic conditions via simulation study.

\section{Methods}

\section{1. feedback gating}

Based on the threshold determined by the Macroscopic Fundamental Diagram (MFD), the strategy of feedback gating control is to control the traffic flow via the gates, which are selected around the boundary of the controlled area. The setting of traffic signals at each gate can be modified accordingly.

According to the MFD, if traffic flow in the controlled area grows beyond the threshold, the exit flow will decrease. Therefore, the inflow should be restricted in order to maximize the throughput of the controlled area. The goal of the controller is to keep the traffic volume of controlled area around the threshold. The logic of feedback control is illustrated in Fig. 1.

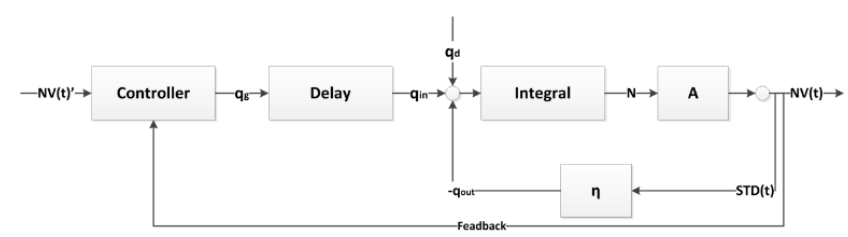

Fig. 1 Regional feedback control logic

$$
\begin{gathered}
\dot{N}=q_{\text {in }}+q_{d}-q_{\text {out }} \\
q_{\text {in }}=q_{g}(t-\tau) ;
\end{gathered}
$$

Where, $q_{\text {in }}$ represents part of traffic flows that entered the controlled area; $q_{d}$ represents inflows at sections without detectors; $q_{\text {out }}$ is the exit flow of controlled area; 


\subsection{MFD of the study area}

Macroscopic Fundamental Diagram (MFD) was first proposed by Godfrey[12] at 1969. The fundamental diagram of traffic flow is a diagram that gives a relation between traffic flow and traffic density. It was found that the exit flow maintains when the inflow grows beyond certain limits based on the simulation results [13]. Geroliminis and Daganzo obtained the MFD of traffic flow and density based on the real data in Japan and simulated data in San Francisco [14], and found that the shape of MFD are affected not by the locations of detectors but by the characteristics of the road network. The curve of the MFD may depend on the traffic demand, however it may be quite stable from day to day [15][16]. Therefore, MFD is the key factor to derive the threshold that maximizes the throughput of the controlled area.

The following equations are adopted to obtain MFD. The Net Volume (NV in veh per $\mathrm{h}$ ) reflects the horizontal axis of MFD, while the Selected Travelled Distance (STD in veh-km per h) reflects the vertical axis of MFD.

$$
\begin{gathered}
N V(k)=\sum_{i \in m} V_{i}(k) \\
S T D(k)=\sum_{i \in n} q_{i}(k) \cdot L_{i}
\end{gathered}
$$

Where $i$ is the link where detectors are distributed; $m$ is the set of links; $k=0,1,2, \ldots$ is the time intervals; $V_{i}(k)$ is the estimated traffic volumes in link $i$ during cycle $k ; n$ is the set of links where detectors are distributed; $q_{i}(k)$ is the measured traffic flow in link $i$

during cycle $k$; and $L_{i}$ is the length of link $i$.The NV and STD are obtained from the link measurements.

Fig. 2 indicates that a macroscopic fundamental diagram shape (inverse $U$ shape) appears during the simulation period with a quite moderate scatter via different replications. It can be seen in Fig. 2 that the STD value increased with increase of NV during the free-flow period, and STD reached the maximum value in a $\mathrm{NV}$ region of 18000 to 19000 veh per h. Then the STD value decreased with the increase of NV beyond the threshold.

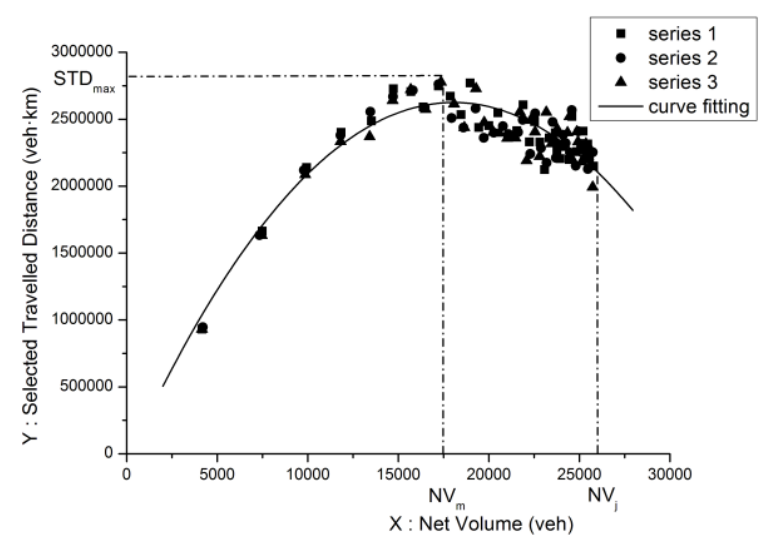

Fig. 2 The MFD of Road Network

\subsection{Identification of key nodes}

It is easy to obtain the appropriate information of each link by simulation. However, it is unrealistic to distribute the detectors on every link of road network due to the limitation of funding and complexity of maintenance. Therefore, the location and numbers of detectors are important. A better MFD can be obtained if the detectors were distributed on the key links.

Some researchers believe that the relationship between nodes and traffic flow can be described in term of power law distribution [17]. This means that a small number of nodes and sections bear the main road traffic volume. The malfunction of a few key nodes and important sections often lead to a large area of congestion and collapse of the entire road network. Therefore, the detectors are laid on the key nodes and important sections in this study.

A novel method based on OD distribution of trips and connectivity of nodes is adopted in this study [18]. This algorithm extracts statistical information of routes and O-D information of trips from trajectories dataset. Three concepts: trip load, route popularity and node criticality, are introduced in developing a tripartite graph modeled interrelationship among the nodes consists of trip, route and intersection. The relation weight matrix of this tripartite graph can be decided by the proportion of routes and level of intersection. That means, the path which is selected by high load trips with higher probability would be more popular, and the intersection which is passed through by more popular paths would be more critical, and vice versa. Taking relevance between node and O-D distribution into account, which can identify network-wide 
key nodes accurately, this algorithm incorporates both topological structure and traffic characteristics. The ratings of trips, paths and intersections can be calcuated via interative process based on the tripartite graph.

\subsection{Section traffic prediction with no detector}

Due to physical constraints, we can't guarantee that all sections in the area are equipped with detectors, and therefore mathematical statistics analysis is required to obtain the data of sections without detector for further analysis.

As shown in Fig.3, the virtual detector is denoted as $X$, and the real detectors are denoted as $X_{1}, X_{2}, \ldots, X_{6}$, which have direct relation with the virtual one. In this study, we consider the volume recorded by virtual detector is only related to the data collected in the vicinity area.

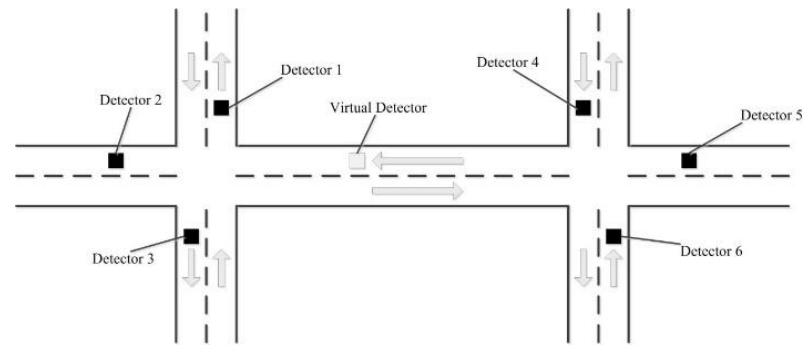

Fig.3 Relationships between virtual and real detectors Artificial neural networks (ANNs) employ a massive interconnection of simple processing elements that incrementally learn from their environment to capture essential linear and nonlinear trends in complex data, so that it provides reliable predictions for new situations containing even noisy and partial information[19]. ANN (Artificial Neural Network) has a strong adaptability, fault tolerance and self-learning ability, and can fit arbitrarily complex nonlinear models to multidimensional data to any desired accuracy [20]. In order to better predict the traffic flow of those sections without detectors, the BP neural network (Back Propagation Neural Network) was adopted, and massive simulation data were used.

The BPN consists of an input layer, an output layer, and one or more hidden layers. The primary structure of the proposed BP neural model was shown in Figure 4. The number of neurons in the input layer was 7 and the output layer consisted of one neuron. The number of hidden layer was 1 . The variables of input neutrons were the signal status of downstream intersection, the green split of downstream intersection, the signal status of upstream intersection, the green split of upstream intersection, the volume of upstream real detector4, the volume of upstream real detector5, and the volume of upstream real detector6. The output neutrons was the traffic flow of virtual detector.

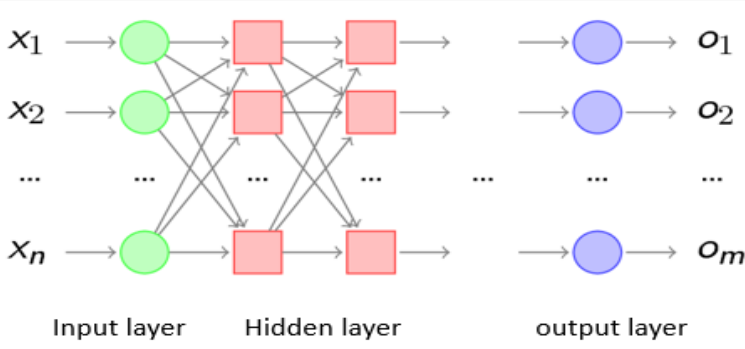

Fig.4 Primary structure of the proposed network

The input data for model training were from the simulation. Multiple simulations were conducted, while the input data were recorded. The best solution was achieved in 1500 epoch. Acceleration was large enough to make the search path oscillate around the minimum indefinitely. The Prediction Model Accuracy was 0.817 .

\section{Simulation study}

\subsection{Study area}

In this study, the area around the Nanning International Exhibition Center is considered as the controlled region to simulate and demonstrate the Regional Traffic Volume Dynamic Control technology. The China-ASEAN Expo is convened in this area every year, and a large number of state organs and financial institutions are located in this region, which contributes to traffic congestion especially during the rush hour. Therefore, the congestion phenomena are remarkable in this region, which will directly affect traffic conditions of surrounding roads and cause a wide range of traffic jams. 40 intersections are included in this study area, which are shown in figure 6 .

\subsection{Definition of gates}

13 intersections are selected as the controlled "gates" at the boundary of controlled region. The traffic volumes can be restricted at these "gates" and the controlled region can be protected during peak period. The locations of the "gates" are indicated in Fig. 5. 


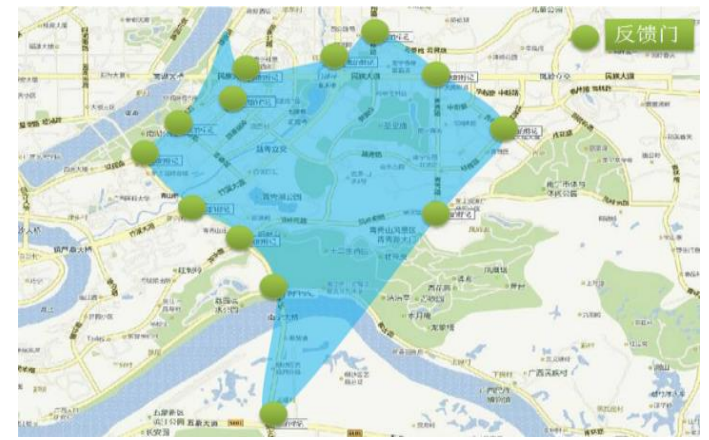

Fig. 5: Demonstration of study area and "gates"

\subsection{MFD of the controlled area}

Two scenarios are designed to compare the MFD shape between the situations in which the detectors are deployed in each section and the detectors are applied only in key nodes. Multiple replications with different random seeds are carried out for each scenario. The comparison results are shown in Fig. 6. Scenario 1 represents the case that detectors are deployed in each section, and scenario 2 represents the case that detectors are applied only in the key nodes. It is clear that the MFD (inverse-U) shape is indeed occurring in both scenarios. The throughput and efficiency of controlled area increases with the increase of $\mathrm{NV}$ before $\mathrm{NV}$ reaches $\mathrm{NV}_{\mathrm{m}}$, and then, the throughput degrades when the traffic flow reaches threshold. To maximize the throughput, the NV should be maintained in the optimal range. The $\mathrm{NV}_{\mathrm{m}}$, which are the critical threshold of control, of two sets of data are very close. That means the accurate control threshold can be obtained even though detectors are deployed only in the key nodes. The curve is fitted based on the scatters. It is found that quadratic equation curve provides a good fitting to the scattered point. The coefficient determination is over $85 \%$.

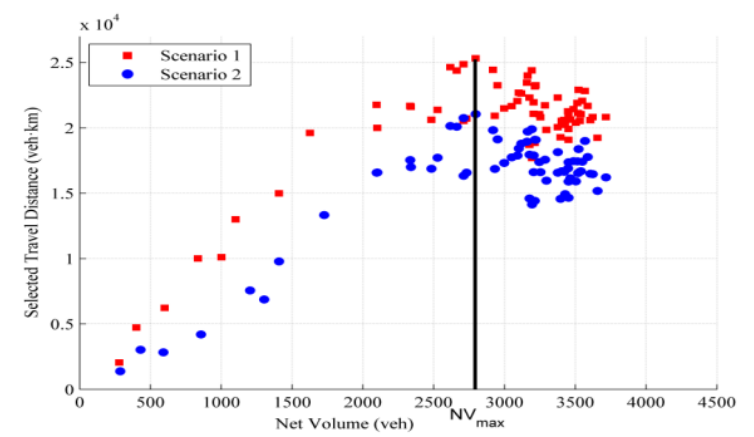

Fig. 6 The comparison of MFD between two scenarios

\subsection{Simulation results}

Two scenarios are designed to compare the traffic conditions between before and after control. The fixed-time signal control is used in Scenarios 1. The gating control logic is implemented in scenario 2, and the threshold of control is set according to the Fig. 6. The gating is activated when NV exceeds the threshold. Because the prediction without detector information would involve additional computation burden, the control interval of the model was set to be 3 minutes. The comparison results are illustrated in Fig. 7 and Table 1, from which the improvements are significant. It is clear in Fig. 7(a) that inflows are controlled to maintain the NV around $\mathrm{NV}_{\mathrm{m}}$ when $\mathrm{NV}$ reaches the threshold and gating control is switched on around $t=15 \mathrm{~min}$. And STD stays at high level after the gating control is switched on, compared to scenario 1 without gating control (Fig. 7(b)). Also, the speed of each section shows a significant improvement in Table 1. An average speed increase of $11.96 \%$ results for the whole network and some sections even get almost $50 \%$ improvements, in contrast to the case without gating control.

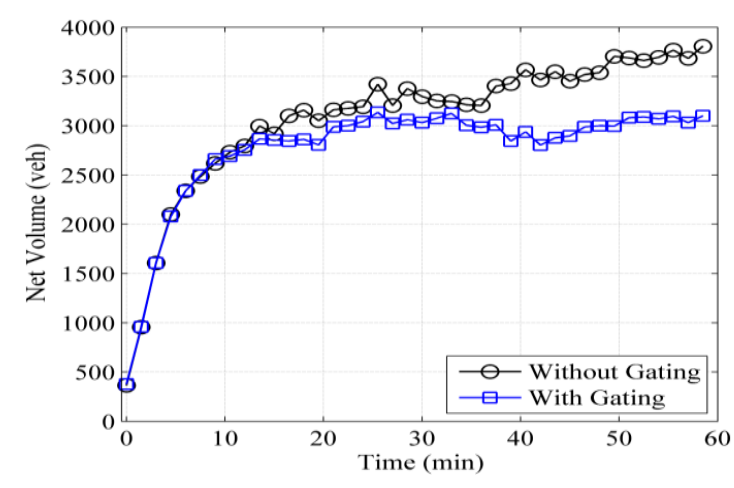

(a)

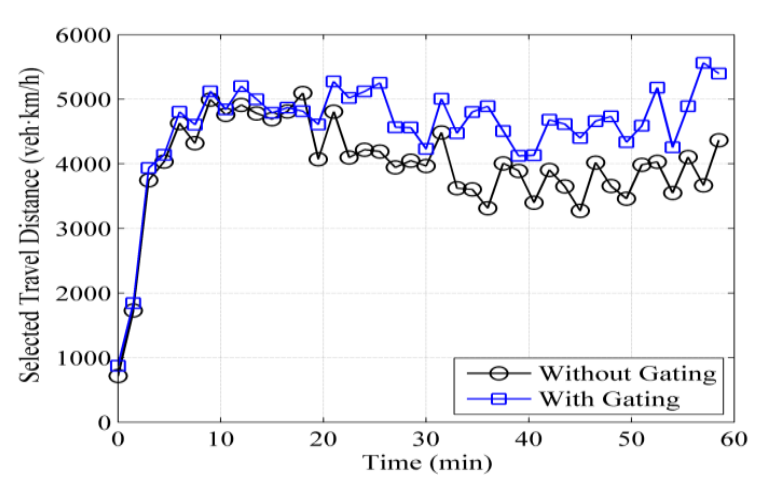

(b)

Fig. 7 (a)The comparison of NV between with- and without- gating; (b) The comparison of STD between with- and without- gating 
Table 1: Comparison between non-gating and gating cases

\begin{tabular}{llllllll}
\hline Speed/Detector ID & 251183 & 178865 & 178853 & 178864 & 147056 & 147055 & Average \\
\hline Before control & 46.87 & 7.78 & 16.47 & 12.24 & 9.79 & 17.58 & 18.14 \\
After control & 49.18 & 11.59 & 23.91 & 14.02 & 11.06 & 18.41 & 20.31 \\
Improve $(\%)$ & 4.93 & 48.98 & 45.17 & 14.54 & 12.97 & 4.72 & 11.96 \\
\hline
\end{tabular}

\section{Conclusion}

An improved dynamic regional traffic volume control strategy is proposed based on the feedback gating and MFD concept. The proposed methodology is applied to the area around the Nanning International Exhibition Center. The key nodes are identified based on OD distribution of trips and connectivity of nodes. The microscopic traffic simulation model is employed as the test platform. The results show that the accurate control

[1]T. YANG, J.K. CHEN, H. YU. Research on transportation capacity of city center area. Urban transport of China. 2003, 1(1): 13-18.

[2]C. F. DAGANZO. The nature of freeway gridlock and how to prevent it. Transportation and traffic theory. 1996. [3]A. de Palma, M. Ben-Akiva, C. Lefevre, and N. Litinsa. Stochastic equilibrium model of peak period traffic congestion. Transportation Science, 17(253-274), 1983.

[4]L.L. ZANG. Optimization of Urban Traffic Signals. Shan Dong University. 2007.

[5]Z.L. Li, X.H. ZHAO. Coordinated Control of Area Traffic Signal Based on Agent. 32(1): 130-133, 2008.

[6]M. Wiering, J. Vreeken, J. Van Veenen. Simulation and optimization of traffic in a city. Intelligent Vehicles Symposium, 453-458, 2004.

[ 7 ]H. ZHANG, Y.Z. CHEN, Y.Z. YANG. Urban Traffic Coordination Control System Based on Multi-Agent. Computer and Communications. 24(2): 94-98, 2006.

[8]Y. LIN, Z.X. XU, S.B. LI. Simulation Optimization of Traffic Signal Control Parameters. Journal Of Transportation Systems Engineering and Information Technology. 10(3): 42-49, 2010.

[9]Y.X. YU, G.Y. JIANG. Disscussion on dynamic dispersal strategy for urban traffic congestion. Modem Transportation Technology. 6(4):90-93, 2009.

[10]R. W. Denney, L. Head and K. Spencer, Signal timing undersaturated conditions, Tech. Rep. FHWA-HOP-09-008 [R], U.S. Department of Transportation, Federal Highway Administration, Washington, DC, USA, 2008. threshold can be obtained even detectors are deployed only in the key nodes. The application of feedback control strategy can improve the efficiency and throughput significantly compared to the case without gating control.

\section{Acknowledgement}

The research is supported by The 863 program with grant number 2012AA063303 and China Postdoctoral Science Foundation with grant number 2013M540102.

[11]Mehdi Keyvan Ekbatani, Anastasios Kouvelas, Ioannis Papamichail \& Markos Papageorgiou. Congestion Control in Urban Networks via Feedback Gating Procedia-Social and Behavioural Sciences. 48:1599-1610, 2012.

[12]J.W. Godfrey. The mechanism of a road network. Traffic Engineering and Control, Vol. 11, 323-327, 1969.

[13]Y.Y. MA. Research on the signal control systems based on the sub-network coordination. Tongji University. 2010.

[ 14 ]N. Geroliminis and C.F. Daganzo. Existence of urban-scale macroscopic fundamental diagrams: some experimental findings. Transportation Research Part B, Vol. 42, pp. 756-770, 2008.

[15]Y. Ji, W. Daamen, S.,Hoogendoorn, S. Laser and X. Qian. Investigating the shape of the macroscopic fundamental diagram using simulation data. Transportation Research Record, 2161, pp. 40-48, 2010.

[16]N. Geroliminis and J. Sun. Properties of a well-defined macroscopic fundamental diagram for urban traffic. Transportation Research Part B, Vol. 45, 605-617, 2011.

[17]J.J. WU, Z.Y. GAO, H.J. SUN..Urban transport system complexity - complex network method and its application. Science Press. 2010.

[18]M. Xu, J.P. Wu, Y.M. Du. A Method of Key Node Ranking for Road Network Based on Tripartite Graph. Journal of Beijing University of Posts and Telecommunications. 37, pp51-54, 2014

[19]Siami-Irdemoosa, Elnaz, Dindarloo, SR. Prediction of fuel consumption of mining dump trucks: A neural networks approach. Applied Energy. 04:77-84, 2015.

[20]Smith M. Neural networks for statistical modeling. International Thompson Computer Press; 1996. 